\title{
AN ELECTRON MICROSCOPE STUDY OF A TAIL ABNORMALITY IN SPERMATOZOA FROM A SUBFERTILE MAN
}

\author{
A. ROSS, SHEILA CHRISTIE AND M. G. KERR* \\ M.R.C. Clinical and Population Cytogenetics Unit, Western General Hospital, \\ Crewe Road, Edinburgh 4, and Department of Obstetrics and Gynaecology, \\ University of Edinburgh, 39 Chalmers Street, Edinburgh
}

(Received 4th March 1970)

\begin{abstract}
Summary. A 38-year-old male with a history of 11 years' involuntary infertility was investigated at a subfertility clinic. Seminal analysis showed a sperm density of 40 to $50 \times 10^{6}$ spermatozoa $/ \mathrm{ml}$ and 1 to $2 \%$ motility. He had a normal male complement of 46 chromosomes and meiotic analysis showed 23 normal bivalents. The testes appeared normal histologically, but in the biopsy specimen abnormal spermatozoa were detected in the seminiferous tubules by electron microscopy. Electron microscope examination showed that $97 \%$ of ejaculated spermatozoa were structurally abnormal, with elongation of the mid-piece, absence of an outer sheath on the main-piece and extra axial fibres.
\end{abstract}

\section{INTRODUCTION}

Despite the difficulties in the classification of sperm abnormalities, there are a few reported cases of subfertility which appear to be due to a single specific defect which dominates the sperm population. Hancock \& Rollinson (1949) described a "decapitated sperm defect" in Guernsey bulls where 95 to $100 \%$ of the sperm heads were detached from their tails. Blom (1966) reported a hereditary defect in the sperm tail of two Jersey bulls, 40 to $50 \%$ of the sperm tails being tightly coiled and non-motile. Ultrastructural studies of these spermatozoa showed that three to five of the inner nine doublet fibres were missing (Blom \& Birch-Andersen, 1966). This anomaly appeared to arise within the epididymis, since it was not demonstrable in testicular spermatozoa. Blom (1968) has also described a "pseudo-droplet" defect in the mid-piece of 7 to $26 \%$ of the spermatozoa in five Friesian bulls. Electron microscopy showed this structure to be a cluster of granules surrounded by one or more layers of mitochondria (Blom \& Birch-Andersen, 1968). The same author described a "corkscrew sperm defect" in bulls of the RDM breed (Blom, 1959), characterized by irregular distribution of the mitochondria within the mid-piece. This is an acquired defect associated with reduced sperm motility and subfertility. In one

* Present address: Department of Obstetrics and Gynaecology. McMaster University, Hamilton Ontario, Canada. 
sterile Friesian bull, $100 \%$ of the spermatozoa showed this defect (Smidt, 1965).

Other specific abnormalities involve the sperm head, e.g. the "knobbed sperm" defect in bulls (Blom \& Birch-Andersen, 1962), "pouch formations" in the nucleus of both bulls and pigs (Bane \& Nicander, 1965), acrosome abnormalities in boar spermatozoa (Bane \& Nicander, 1966) and mice (Rajasekarasetty, 1954) and coarse granulation of nuclear chromatin in bulls (McCosker, 1969).

There are two reports describing comparable structural abnormalities in human spermatozoa. Williams (1950) described a type of "centriolar-mitochondrial" sperm defect which involved the entire sperm population and caused absolute sterility. It was characterized by prolongation of the midpiece and total absence of motility; in most instances the spermatozoan mainpiece was completely absent, but occasionally the tail became doubled back on itself and the end-piece was seen protruding from the neck region. Kagan (1963) demonstrated that the nuclear chromatin was coarse in some patients with oligospermia suggesting that maturation had not been completed at the time of ejaculation. In some cases of necrospermia, the axial filament complex was either destroyed or absent, with consequent loss of motility.

This report describes the ultrastructural appearances of a tail defect which involves $97 \%$ of spermatozoa in a subfertile man. This 38-year-old patient had experienced 11 years' involuntary infertility. Clinical examination of his wife showed no explanation for her failure to conceive. Examination of the husband revealed no physical abnormalities.

\section{MATERIAL AND METHODS}

Peripheral blood and buccal smears were taken to determine the chromosome complement and sex chromatin status. A testicular biopsy was performed and the biopsy material was subjected to meiotic chromosome analysis, histology and electron microscopy. Seminal analysis was carried out on three fresh ejaculates over a period of 2 months; this included estimates of motility, sperm density and morphology. The remainder of the samples were processed in the following manner for electron microscopy.

Within 15 min after ejaculation, the seminal fluid was divided into two equal parts. One part was centrifuged at $3000 \mathrm{rev} / \mathrm{min}$ for $15 \mathrm{~min}$, the supernatant was removed and then replaced with $5 \%$ glutaraldehyde in cacodylate buffer. After $1 \mathrm{hr}$, the fixative was removed, the pellet broken into smaller pieces, washed in buffer, post-fixed in $1 \%$ osmium tetroxide, processed through upgraded alcohols into epoxy propane and finally embedded in Araldite and polymerized at $60^{\circ} \mathrm{C}$ for $48 \mathrm{hr}$.

The remainder of the specimen was centrifuged at $3000 \mathrm{rev} / \mathrm{min}$ for $15 \mathrm{~min}$, the supernatant was removed and the spermatozoa were re-suspended in $0.2 \mathrm{M}$ ammonium acetate. This washing procedure was repeated three times, after which the pellet was re-suspended in $0.2 \mathrm{M}$-ammonium acetate. A drop of this suspension was placed on a formvar-coated grid and allowed to dry. The grid was finally coated with a thin layer of carbon and shadowed with gold/ palladium. 
PLATE 1
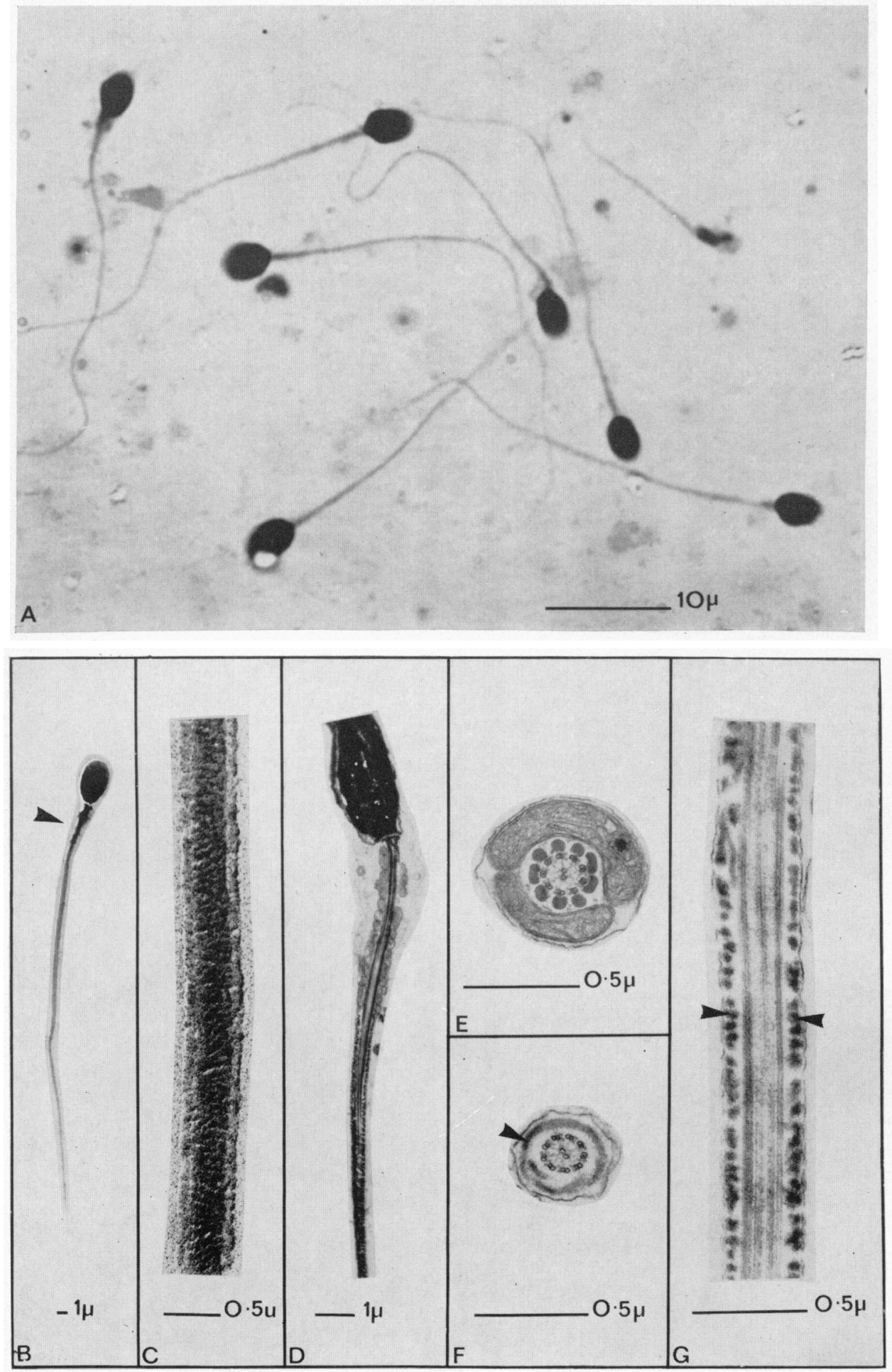
PLATE 2
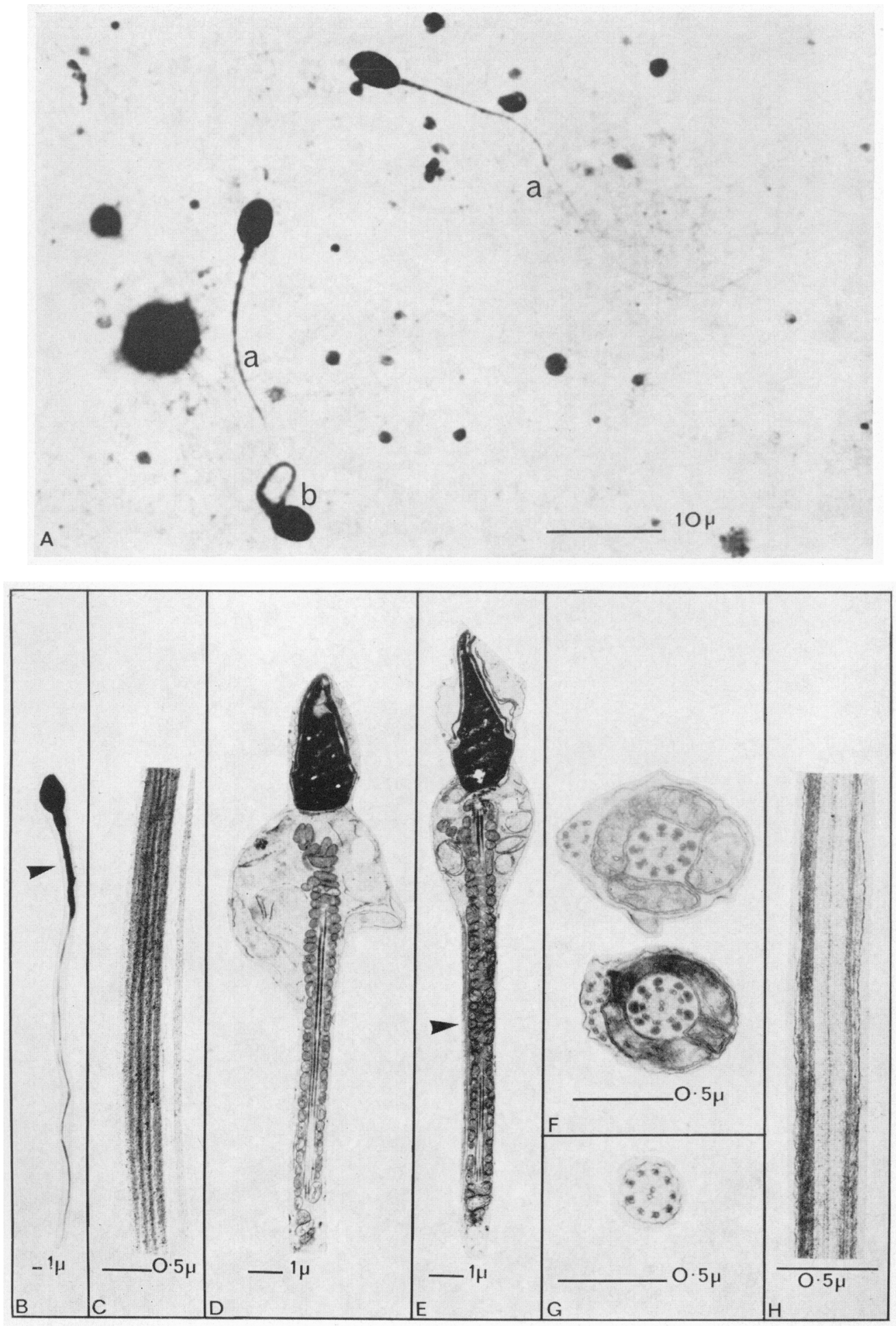
All electron microscope specimens were examined and photographed using an AEI EM6B electron microscope.

\section{RESULTS}

The volume of the ejaculates ranged from 2.9 to $3.2 \mathrm{ml}$ and sperm density from 23 to $50 \times 10^{6}$ spermatozoa $/ \mathrm{ml}$. There was a virtual absence of motility, only 1 to $2 \%$ of the spermatozoa showing any activity. Preliminary light microscopy using haematoxylin and eosin stained preparations suggested that the sperm tails were shorter than normal (cf. Pl. 1, Fig. A and Pl. 2, Fig. A). However, on closer examination the main-piece could be seen to be of roughly normal length but was much finer than normal. With the nigrosin-eosin staining techniques, the very fine filamentous tails were seen more clearly and this technique showed that the mid-piece was longer than normal. A large elongated cytoplasmic droplet was present in 40 to $45 \%$ of spermatozoa and $10 \%$ had an amorphous head.

\section{EXPLANATION OF PLATES \\ PLATE 1}

Normal spermatozoa.

Fic. A. Light microscope photograph of spermatozoa stained by haematoxylin and eosin.

FIG. B. Electron micrograph of a gold/palladium-shadowed spermatozoon. Note the size of the mid-piece which corresponds in length to the head.

Fig. C. Shadowed preparation of the main-piece showing the cross striations of the outer sheath.

Fig. D. Longitudinal section of a spermatozoon showing part of the head, mid-piece and part of the main-piece.

Fig. E. Cross section through the mid-piece with the mitochondria surrounding a $9+9+2$ axial filament complex.

FIG. F. Cross section through the main-piece showing the outer sheath (arrowed) surrounding a $9+2$ axial filament complex.

FIG. G. Longitudinal section through the main-piece showing the appearance of the outer sheath (arrowed) cut in cross section.

\section{PLATE 2}

Abnormal spermatozoa.

Fig. A. Light microscope photograph of abnormal spermatozoa stained with haematoxylin and eosin. Spermatozoa with fine main-pieces (a) and a spermatozoon with the tail folded back (b) on to the mid-piece are shown.

Fig. B. Electron micrograph of a gold/palladium-shadowed spermatozoon, the mid-piece of which (arrowed) is approximately four times the length of the head.

Fic. C. Whole mount shadowed preparation of the main-piece showing an absence of cross striations of the outer sheath.

FIG. D. Longitudinal section through the head and mid-piece. Note the large cytoplasmic droplet and the length of the mid-piece.

FIG. E. Similar to Fig. D but with extra axial filament fibres (arrowed) between the plasma membrane and the mitochondria.

Fig. F. Cross section through the mid-piece showing various numbers of extra axial filament fibres.

FIG. G. Cross section through the main-piece showing the absence of an outer sheath. FIg. H. Longitudinal section through the main-piece showing the absence of the outer sheath. 
The buccal smear was chromatin negative and chromosome analysis on peripheral blood showed a normal male chromosome complement. Meiotic chromosome analysis revealed 23 normal bivalents. Histological examination of the testicular biopsy showed the seminiferous tubules to be solid, with brisk cell division and all stages of spermatogenesis through to mature spermatozoa. The interstitium and Leydig cells appeared normal.

In whole-mount preparations for electron microscopy, the outline of the majority of sperm heads appeared normal. In $97 \%$ of the spermatozoa, the length of the mid-piece exceeded the normal range of 4 to $5 \mu$ (Pl. 1, Fig. B), varying in length between 13 and $17 \mu(\mathrm{Pl}$. 2, Fig. B); the mid-piece also varied in diameter between 0.8 and $1.5 \mu$, which was slightly greater than the normal range of 0.5 to $1 \mu$. The main-piece did not show the normal cross-grained appearance of the outer sheath (Pl. 1, Fig. G), the outer fibres of the axial filament complex being visible (Pl. 2, Fig. C). Despite the elongation of the midpiece, the total length of spermatozoa averaged $50 \mu$, which is the average length of normal human spermatozoa.

Examination of thin sections of sperm heads showed a normal acrosome but there was coarse granulation of the nuclear chromatin in $46 \%$, a feature suggestive of immaturity. Twelve per cent of the cells examined were spermatids at various stages of spermiogenesis.

In the majority of spermatozoa with an elongated mid-piece, the mitochondria were distributed evenly along the axial filament complex as in normal spermatozoa (cf. Pl. 1, Fig. D and Pl. 2, Figs. D and E) but in a small number of spermatozoa, the distribution of mitochondria was irregular. In some longitudinal sections, axial fibres in addition to the normal $9+9+2$ axial filament complex (Pl. 1, Fig. E) were seen between the mitochondria and the plasma membrane (Pl. 2, Fig. E). This defect was seen more clearly in cross section (Pl. 2, Fig. F), where a variable number of fibres could be seen between the mitochondria and the plasma membrane. Approximately $37 \%$ of cross sections through either the mid-piece or the main-piece showed this abnormality. Longitudinal and cross sections of the tail showed an absence of the outer sheath which is seen in normal spermatozoa (cf. Pl. 1, Figs. F and G, and Pl. 2, Figs. $\mathrm{G}$ and $\mathrm{H}$ ). A few tails were seen where the outer sheath was present; in some of these, the mid-piece was elongated and in others it was of normal size.

Electron microscopic examination of spermatozoa within seminiferous tubules in the biopsy specimen showed elongation of the mid-piece and absence of the outer sheath on the main-piece of maturing spermatozoa. No extra axial fibres could be detected.

\section{DISCUSSION}

Some types of sperm defects appear to arise within the epididymis following normal spermiogenesis. In this case, spermatozoa with an elongated mid-piece and absence of the outer sheath could be seen in the testicular biopsy sections, indicating that these defects originated during spermiogenesis within the seminiferous tubules. Extra axial filaments, however, were found in ejaculated spermatozoa but were not seen in the spermatozoa in the seminiferous tubules. 
The abnormality might have resulted from folding of the tail back on itself within the plasma membrane at a later stage in development.

The mid-piece defect is similar to that described by Williams (1950), but he concluded that the tail failed to develop. The spermatozoa described here all seemed to possess a main-piece but this was difficult to see by light microscopy either because it could be folded back along itself, hence the extra axial filaments seen by electron microscopy, or because it appeared as a very fine filament due to the absence of its outer sheath.

\section{ACKNOWLEDGMENTS}

The authors are grateful to Mr Peter Edmond for performing the testicular biopsy, Dr N. Maclean for the histological studies, Dr D. Wooley for his invaluable discussion and advice during the preparation of this paper, Dr Ann Chandley for the meiotic preparations, and Mr A. R. Ross, Mr A. Sutherland, Mrs Lily Gowans and Sister M. Isdale for technical assistance.

This investigation was supported by a grant from the Medical Research Council.

\section{REFERENCES}

BAne, A. \& Nicander, L. (1965) Pouch formation by invagination of the nuclear envelope of bovine and porcine sperm as a sign of disturbed spermiogenesis. Nord. VetMed. 17, 628.

BANE, A.\& NiCANDER, L. (1966) Electron and light microscopical studies of spermateliosis in a boar with acrosome abnormalities. F. Reprod. Fert. 11, 133.

Bцом, E. (1959) A rare sperm abnormality, 'corkscrew sperms' associated with sterility in bulls. Nature, Lond. 183, 1280.

BLom, E. (1966) A new sterilizing and hereditary defect (the Dag defect) located in the bull sperm tail. Nature, Lond. 209, 739.

BLom, E. (1968) A new sperm defect 'pseudodroplets', in the middle piece of the bull sperm. Nord. VetMed. 20, 279.

Blom, E. \& Birch-Andersen, A. (1962) Ultrastructure of the sterilizing 'knobbed sperm' defect in the bull. Nature, Lond. 194, 989.

BLom, E. \& Birch-ANDersen, A. (1966) The ultrastructure of a new hereditary sterilizing defect (the Dag defect) in the bull sperm tail. Proc. Vth Wld Congr. Fert. Steril., Stockholm. Excerpta med. Internat. Congr. Series No. 132, p. 602.

Blom, E. \& Brrah-Andersen, A. (1968) The ultrastructure of the "pseudo-droplet" defect in the bull sperm. Proc. VIth Int. Congr. Anim. Reprod. AI, Paris, $1,117$.

Hancock, J. L. \& Rollinson, D. H. L. (1949) A seminal defect associated with sterility in Guernsey bulls. Vet. Rec. 61, 742.

KAGAN, C. A. (1963) A study of the ultrastructure of human spermatozoa in oligospermia and necrospermia. Urologica, 28, 34.

MCCoskeR, P. J. (1969) Abnormal spermatozoan chromatin in infertile bulls. F. Reprod. Fert. 18, 363.

RAJASEKARASETTY, M. R. (1954) Studies on a new type of genetically-determined quasisterility in the house mouse. Fert. Steril. 5, 68.

SMmo, W. J. (1965) Afwijkingen wan het niddenstuk bij stierespermien. Tijdschr. Diergeneesk. 90, 1613.

Wirliams, W. W. (1950) Male sterility due to centriolor-mitochondrial disease of the spermatozoa. 7. Urol. 64, 614 . 